\title{
Stellar Orbital Studies in Normal Spiral Galaxies I: Restrictions to the Pitch Angle
}

\author{
A. Pérez-Villegas, B. Pichardo, E. Moreno \\ Instituto de Astronomía, Universidad Nacional Autónoma de México, A.P. 70-264, 04510, \\ México, D.F.; Universitaria, D.F., México; \\ barbara@astro.unam.mx
}

\begin{abstract}
We built a family of non-axisymmetric potential models for normal non-barred or weakly-barred spiral galaxies as defined in the simplest classification of galaxies: the Hubble sequence. For this purpose a three-dimensional self-gravitating model for spiral arms PERLAS is superimposed to the galactic axisymmetric potentials. We analyze the stellar dynamics varying only the pitch angle of the spiral arms, from $4^{\circ}$ to $40^{\circ}$, for an Sa galaxy, from $8^{\circ}$ to $45^{\circ}$, for an Sb galaxy, and from $10^{\circ}$ to $60^{\circ}$, for an Sc galaxy. Self-consistency is indirectly tested through periodic orbital analysis, and through density response studies for each morphological type. Based on ordered behavior, periodic orbits studies show that for pitch angles up to approximately $15^{\circ}, 18^{\circ}$, and $20^{\circ}$ for $\mathrm{Sa}, \mathrm{Sb}$ and $\mathrm{Sc}$ galaxies, respectively, the density response supports the spiral arms potential, a requisite for the existence of a long-lasting large-scale spiral structure. Beyond those limits, the density response tends to "avoid" the potential imposed by mantaining lower pitch angles in the density response; in that case the spiral arms may be explained as transient features rather than long-lasting large-scale structures. In a second limit, from a phase space orbital study based on chaotic behavior, we found that for pitch angles larger than $\sim 30^{\circ}, \sim 40^{\circ}$ and $\sim 50^{\circ}$ for $\mathrm{Sa}, \mathrm{Sb}$, and Sc galaxies, respectively, chaotic orbits dominate all phase space prograde region that surrounds the periodic orbits sculpting the spiral arms and even destroying them. This result seems to be in good agreement with observations of pitch angles in typical isolated normal spiral galaxies.
\end{abstract}

Subject headings: Chaos — galaxies: evolution — galaxies: kinematics and dynamics — galaxies: spiral — galaxies: structure 


\section{Introduction}

When Hubble $(1926,1936)$ introduced his classification scheme of galaxies, he emphasized that the turning-fork diagram he obtained, depicted the systematic variation of the morphological characteristics, and the terms "early type" and "late type" referred only to the relative position of a galaxy in this empirical sequence. The sequence did not imply temporal evolution connections. Some modifications have been introduced to this scheme over the years (de Vaucouleurs 1959; Sandage 1961). In the case of isolated galaxies, astronomers keep searching in this well-ordered sequence of galaxy types for a clue to possible formation and evolutionary processes to explain galactic morphology. It is not surprising then, that morphology is frequently an underlying theme in the study of galaxies. A few percent of all galaxies are unclassifiable, many of these due to their unusual morphology produced by their interacting nature. In this paper we focus on intrinsic dynamical processes of galaxies, this refers to relatively isolated galaxies.

Spiral galaxies are classified in the Hubble sequence based mainly on two criteria: the pitch angle and the bulge to disk luminosity ratio. On Sandage's classification (Sandage 1975), three parameters to classify spiral galaxies are employed: the bulge to disk ratio, the pitch angle of the spiral arms, and the arms fragmentation into stars. These two classifications are very similar. Then, early type spirals show small pitch angles with smooth structure and conspicuous central bulges. For late spirals, the arms are open and flocculent, and the central bulges are smaller. Finally, Sb galaxies are between the above two types.

Since the Hubble classification was introduced, astronomers are puzzled and searching correlations or dependences between galactic parameters and morphological types. Holmberg (1958) and Sérsic (1987), compiled and analyzed photometric data (integrated magnitudes, colors and diameters) for hundreds of galaxies, and found a correlation between the Hubble type and galaxy colors. The mass ratio $M_{H_{2}} / M_{\text {Tot }}$ decreases with type from Sa to Sd (Sage 1993; Roberts \& Haynes 1994). The total mass decreased as the Hubble type varied from Sa to Sc (Pişmiş \& Maupomé 1978; Maupomé, Pişmiş \& Aguilar 1981; Roberts \& Haynes 1994). Another interesting correlation is found in the maximum value of rotation velocity: values for Sa galaxies are higher than Sc galaxies (Rubin et al. 1985; Sandage 2000; Sofue \& Rubin 2001); although, the maximum rotation velocity presents a large scatter. Along the Hubble sequence, spiral galaxies disks tend to be thinner (Ma 2002). Some correlations are also found with the pitch angle, like the one related with the central supermassive black hole in spiral galaxies whose mass seems to decrease with the pitch angle (Seigar et al. 2008; Shields et al. 2010); spiral galaxies with higher rotational velocity also have tighter spirals (Kennicutt 1981; Savchenko \& Reshetnikov 2011); in the same manner, it seems that for disks with lower surface densities and lower total mass-luminosity ratios, pitch angles tend 
to be larger (Ma 2002).

Regarding specifically to spiral arms morphology, studies of this type in galaxies, started earlier than the Hubble classification. Von der Pahlen (1911), Groot (1925), Danver (1942), and Kennicutt (1981) found that spiral arms in galaxies are well fitted with logarithmic helices. Kennicutt (1981) concluded that the pitch angle in early type galaxies tends to be smaller than in late type galaxies, but there is a large scatter in the pitch angle within each morphological type, concluding that the ideal Hubble classification is not closely followed by real galaxies (Kennicutt 1981; Ma et al. 2000; Davis et al. 2012).

In the Lin \& Shu (1964) spiral density wave theory, galactic spiral arms are modeled as a periodic perturbation to the axisymmetric background disk's potential. This is known as the tight-winding approximation (TWA) for small pitch angles, or WKB approximation (Wentzel-Kramers-Brillouin approximation of quantum mechanics -Binney \& Tremaine 1994). The solution for bisymmetric spiral arms provided by the TWA takes the form,

$$
\Phi(R, \phi)=f(R) \cos [2 \phi+g(R)] .
$$

where the function $f(R)$ is the amplitude of the perturbation, and $g(R)$ represents the geometry of the spiral pattern. The amplitude function $f(R)$ given by Contopoulos \& Grosbøl (1986) and $g(R)$ of the form presented by Roberts, Huntley \& van Albada (1979) are commonly used. Most orbital studies in spiral galaxies theory, have employed a spiral potential of the form in equation 1. However, the majority of disk galaxies possesses strong spiral structures, making clear that this large scale structure is an important non-axisymmetric component of galaxies that deserves an effort to model it beyond a simple perturbing term. In this work we have chosen a more physically and observationally motivated model of the spiral arms for disk galaxies, the one called PERLAS from Pichardo et al. (2003), based on a three-dimensional model mass distribution, which allows a more detailed representation of spiral arms.

Concerning to large scale structures in galaxies, these seem to be related mainly to ordered orbital behavior (Patsis 2008). This is the case for example, of grand design galaxies, where non-axisymmetric structures are composed by material librating around the family of periodic orbits known as $\mathrm{X}_{1}$ (Contopoulos 2002). In spiral arms, where the $\mathrm{X}_{1}$ periodic orbits dominate, the flow of material through the arms can be described as a "precessing ellipses" flow. This is supported by many observational and theoretical studies (Patsis, Contopoulos \& Grosbøl 1991). Spiral galaxies, with their thin disks and smooth spiral arms may seem to be dominated by ordered motion, but deeper orbital studies show that chaos may be significant in spiral galaxies (Contopoulos 1983,1995; Contopoulos et al. 1987; Grosbøl 2003; 
Voglis, Stauropoulos \& Kalaptharakos 2006; Contopoulos \& Patsis 2008; Patsis et al. 2009), and that chaotic orbits surrounding stable periodic orbits could also support the spiral arms. However, spiral arms and bars, for example, are not expected to originate out of only chaotic orbits. Patsis \& Kalapotharakos (2011) call ordered spirals those that have as building block a set of stable periodic orbits; on the other hand, they call chaotic spirals those that they believe are constituted mainly from stars in chaotic motion.

Currently, discussion about the nature of spiral arms as a long-lasting or transient structure is ongoing in the field. Theoretical studies have demonstrated that spiral arms might be rather transient structures (Goldreich \& Lynden-Bell 1965; Julian \& Toomre 1966; Sellwood \& Carlberg 1984; Foyle et al. 2011; Sellwood 2011; Pérez-Villegas et al. 2012; Fujii \& Baba 2012; Kawata et al. 2012). However, if spiral arms are smooth, weak and/or with small pitch angles, the spiral structure could be long-lasting (Pérez-Villegas et al. 2012, that study is referred to late type spirals).

In a previous paper (Pérez-Villegas et al. 2012), we found two clear limits for the pitch angle in late (Sc) spiral galaxies, one that sets a maximum limit for which steady spiral arms are plausible (based on periodic orbital studies), beyond which, a transient nature for the arms is proposed; the second limit is for which spiral arms are so opened that orbital chaos dominates completely. In this second paper, we extend our studies to early (Sa) and intermedium ( $\mathrm{Sb}$ ) galaxies to see if these limits found in late spirals are also followed by the rest of spirals in the Hubble sequence and if the values of those limits match with observations. We isolate the effect of the pitch angle, that represents the least restricted parameter (i.e. the one with more spread values going from $4^{\circ}$ to $50^{\circ}$ ), and for that reason the one with likely more effect on the stellar dynamics. We employ for this purpose, realistic values for the rest of the parameters that identify approximately typical Sa, Sb and Sc galaxies. The rest of the structural parameters such as angular speed, perturbation strength (spiral arms mass), etc., are better restricted in the sense that the ranges for those values are tighter, measured both by observations and/or by self-consistent models. Specific calculations regarding these parameters (angular speed, spiral arms strength, and axisymmetric components) will be discussed in a forthcoming paper.

This paper is organized as follows. The galactic potential and methodology are described in Section 2, Our results: two pitch angle restrictions in galaxies, the first based on ordered orbital behavior, and the second based on chaotic behavior, are presented in Section 3 , Finally, we present a discussion and our conclusions in Section 4. 


\section{Methodology and Numerical Implementation}

We have constructed a family of models for the potential of normal spiral galaxies (Sa, $\mathrm{Sb}$ and Sc), as classified by Hubble (1926), and also based on recent observational parameters taken from the literature. For this purpose we solved numerically the equations of motion for stars in an axisymmetric potential plus a spiral arm potential. The main methods to study stellar dynamics that we use in this work are periodic orbital analysis and Poincaré diagrams.

\subsection{Models for Normal Spiral Galaxies}

The most common method to model spiral arms is a two-dimensional bisymmetric local potential approximated by a cosine function (based on the solution for the TWA). It assumes that spiral arms are smooth, self-consistent perturbations to the axisymmetric potential. In this regime, self-consistency can not be assured if a little larger pitch angles and/or slightly larger amplitudes to the spiral arms than the ones assumed for the TWA, are imposed.

To model typical spiral arms in galaxies (i.e. pitch angles larger than about $6^{\circ}$, or mass of the spiral arms larger than $1 \%$ of the disk mass), one goes readily far away from the TWA self-consistency limits. Spiral arms in real galaxies, are complicated three-dimensional gigantic structures, far intricate to be approximated with a function as simple as a cosine. To test self-consistency in these type of potentials, we analyzed ordered and chaotic orbital behavior and specifically the construction of periodic orbits. Between a cosine potential and a potential based on mass distribution, the differences are not negligible at all, specially, when we are dealing with chaos.

To this purpose, we employ the spiral arms potential called PERLAS from Pichardo et al. (2003), this potential is formed by individual potentials of oblate inhomogeneous spheroids lying along the logarithmic spiral locus given by Roberts, Huntley, \& van Albada (1979),

$$
g(R)=-\left(\frac{2}{N \tan _{i_{p}}}\right) \ln \left[1+\left(R / R_{s}\right)^{N}\right],
$$

with $i_{p}$ the pitch angle. $R_{s}$ marks the galactocentric position where the spiral arms begin, and $N$ is a constant that gives the shape to the starting region of the spiral arms (we set it to 100; for details see Pichardo et al. 2003).

This bisymmetric self-gravitating potential is more realistic since it is based on a three- 
dimensional density distribution, providing a much more complicated function for the gravitational potential, unlike a two-dimensional local arm like the cosine potential. PERLAS is observationally motivated; comparison with other theoretical models have been already published (Pichardo et al. 2003; Martos et al. 2004; Antoja et al. 2009, 2011). We have tested approximately the self-consistency of the model, through the reinforcement by the stellar orbits (Patsis et al. 1991; Pichardo et al. 2003).

Parameters used to fit normal spiral galaxies ( $\mathrm{Sa}, \mathrm{Sb}$ and $\mathrm{Sc}$ ), with references are presented in Table1. The spiral arms potential is superimposed on an axisymmetric background, described by a massive halo (Allen \& Santillán 1991), and a Miyamoto-Nagai (1975) disk and bulge. We fitted the rotation curve based on observational data from literature of the masses of the disk and bulge vs. disk mass (depending on morphological type). With this information, we derived a halo mass using a typical maximum velocity in the rotation curve for each type. In Figure 1, we show the resulting rotation curves.

Theoretical studies of orbital self-consistency in normal spiral galaxies show that weak spiral arms end at corotation and strong spiral arms end at the 4/1 resonance (Contopoulos \& Grosbøl 1986, 1988; Patsis, Contopoulos \& Grosbøl 1991). Then, the spiral arms limits depend on the position of the Inner Lindblad Resonance (ILR), for the inner part, and corotation for the outer part of the spirals (in our models we place the spiral arms between these two resonances). Consequently, the angular speed of the spiral arms, $\Omega_{p}$, sets the corresponding value of the beginning and the end of these arms. Measuring spiral arms angular velocities observationally is not an easy task. We know however that on average, earlier type galaxies tend to be more massive, therefore Sa galaxies rotate faster than Sc galaxies. On the other hand, considering the few observational studies of angular speeds from literature, these speeds seem to present a small range of difference from Sa to Sc, ranging between 35 and $15 \mathrm{~km} \mathrm{~s}^{-1} \mathrm{kpc}^{-1}$. We employed for the axisymmetric models mass average values for the different components. With a clockwise rotation for the disk, we assume an angular velocity of $\Omega_{p}=-30,-25$, and $-20 \mathrm{~km} \mathrm{~s}^{-1} \mathrm{kpc}^{-1}$, for the spiral arms in Sa, Sb, and Sc galaxies, respectively (see Table 1 for references). In Figure 2 we show resonance diagrams for our galactic models. We take a fairly good approximation to the spiral arms mass, of $3 \%$ of the total disk mass (Pichardo et al. 2003), independently of the Hubble type. Additionally, to assure the spiral mass we fixed to our models is within observational limits, we have employed the parameter $Q_{T}$ (Combes \& Sanders 1981). This parameter has been implemented in studies of bars and spiral arms (Buta \& Block 2001; Laurikainen \& Salo 2002; Buta et al. 2004; Laurikainen et al. 2004; Block et al. 2004; Vorobyov 2006; Kalapotharakos et al. 2010) to measure the strength of large scale non-axisymmetric structures in galaxies. The parameter $Q_{T}$ is defined as 


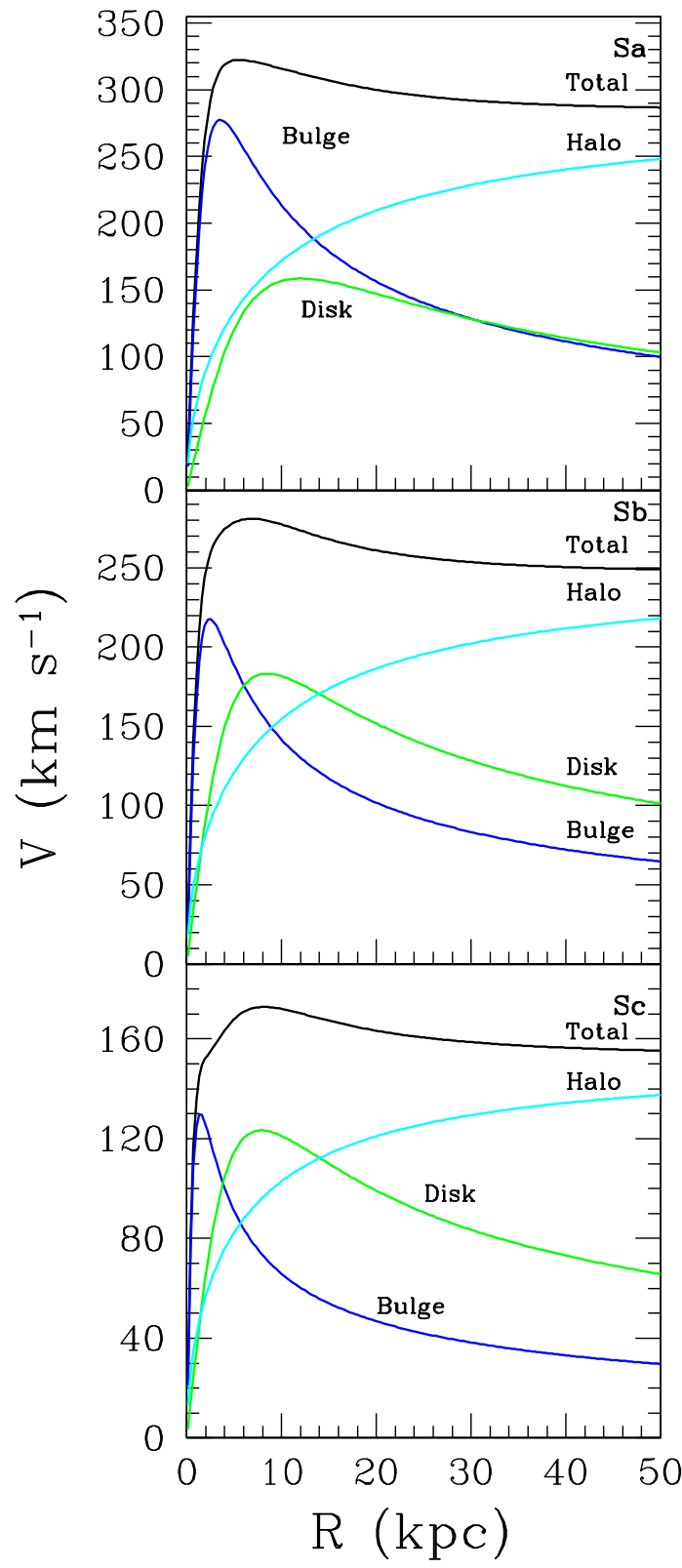

Fig. 1.- Rotation curves for Sa (top panel), Sb (middle panel) and Sc (bottom panel) galaxies. 


$$
Q_{\mathrm{T}}(R)=F_{T}^{\max }(R) /\left|\left\langle F_{\mathrm{R}}(R)\right\rangle\right|,
$$

where $\mathrm{F}_{T}^{\max }(\mathrm{R})=\left|\left(\frac{1}{R} \partial \Phi(\mathrm{R}, \theta) / \partial \theta\right)\right|_{\max }$, represents the maximum amplitude of the tangential force at radius $R$, and $\left\langle F_{R}(R)\right\rangle$, is the mean axisymmetric radial force at the same radius, derived from the $\mathrm{m}=0$ Fourier component of the gravitational potential. In Figure 3 we show an example of the behavior of the parameter $Q_{\mathrm{T}}(R)$ given by equation 3 , for an Sa (solid line), Sb (dotted line) and Sc (dashed line) galaxies. In these three cases we have set the pitch angles to their maximum values permitted before chaos destroys all the periodic orbits support $\left(30^{\circ}, 40^{\circ}, 50^{\circ}\right.$, for $\mathrm{Sa}, \mathrm{Sb}$, and $\mathrm{Sc}$ galaxies, respectively). Note how even for cases with high pitch angles, the parameter $Q_{\mathrm{T}}(R)$ keeps always lower than the observed maximum values in galaxies (Buta et al. 2005). In Figure 4 we present the maximum value of the parameter $Q_{T}$ for each type of galaxy as we increase the pitch angle from $0^{\circ}$ to $90^{\circ}$. Values up to 0.4 for the $Q_{T}$ parameter are consistent with observed spirals (Buta et al. 2005). 


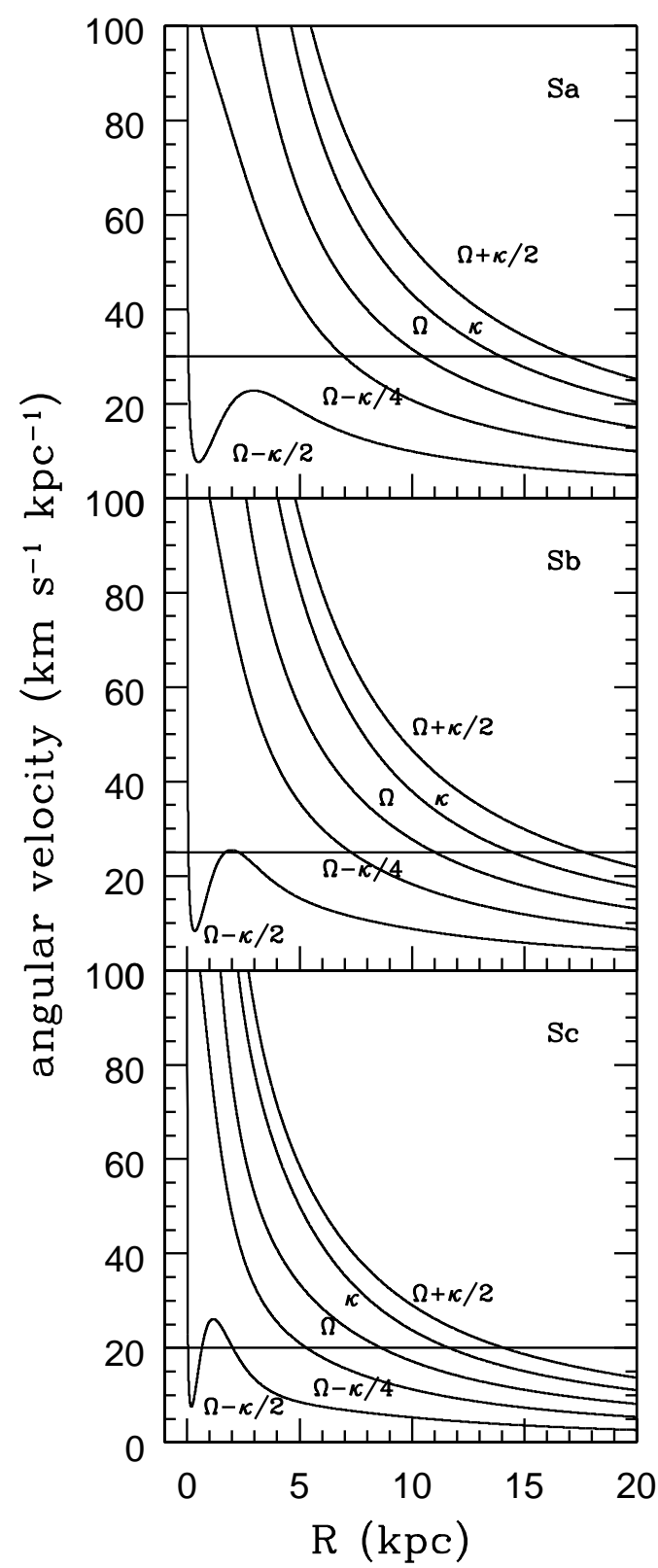

Fig. 2.- Resonance diagrams for $\mathrm{Sa}$ (top panel), Sb (middle panel) and Sc (bottom panel) galaxies. Horizontal lines denote the spiral pattern angular speed: $\left|\Omega_{p}\right|=$ 30,25 and $20 \mathrm{~km} \mathrm{~s}^{-1} \mathrm{kpc}^{-1}$ for an Sa, an $\mathrm{Sb}$ and an Sc, respectively. 


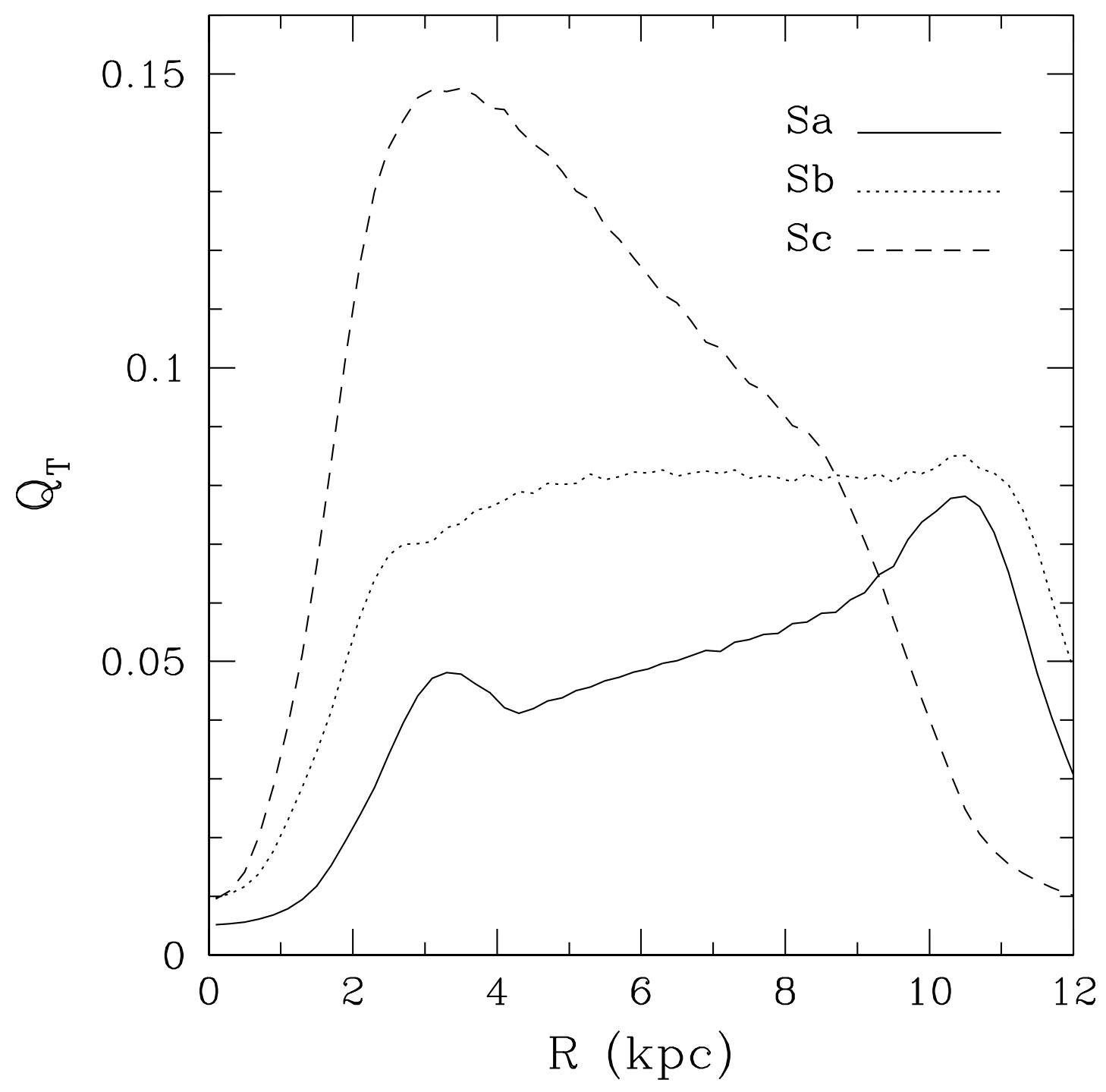

Fig. 3.- $Q_{T}$ parameter (equation 3) for an Sa (solid line), Sb (dotted line), and Sc (dashed line) galaxy, where the pitch angles are $30^{\circ}, 40^{\circ}$ and $50^{\circ}$ for an $\mathrm{Sa}, \mathrm{Sb}$ and $\mathrm{Sc}$ galaxy, respectively. 


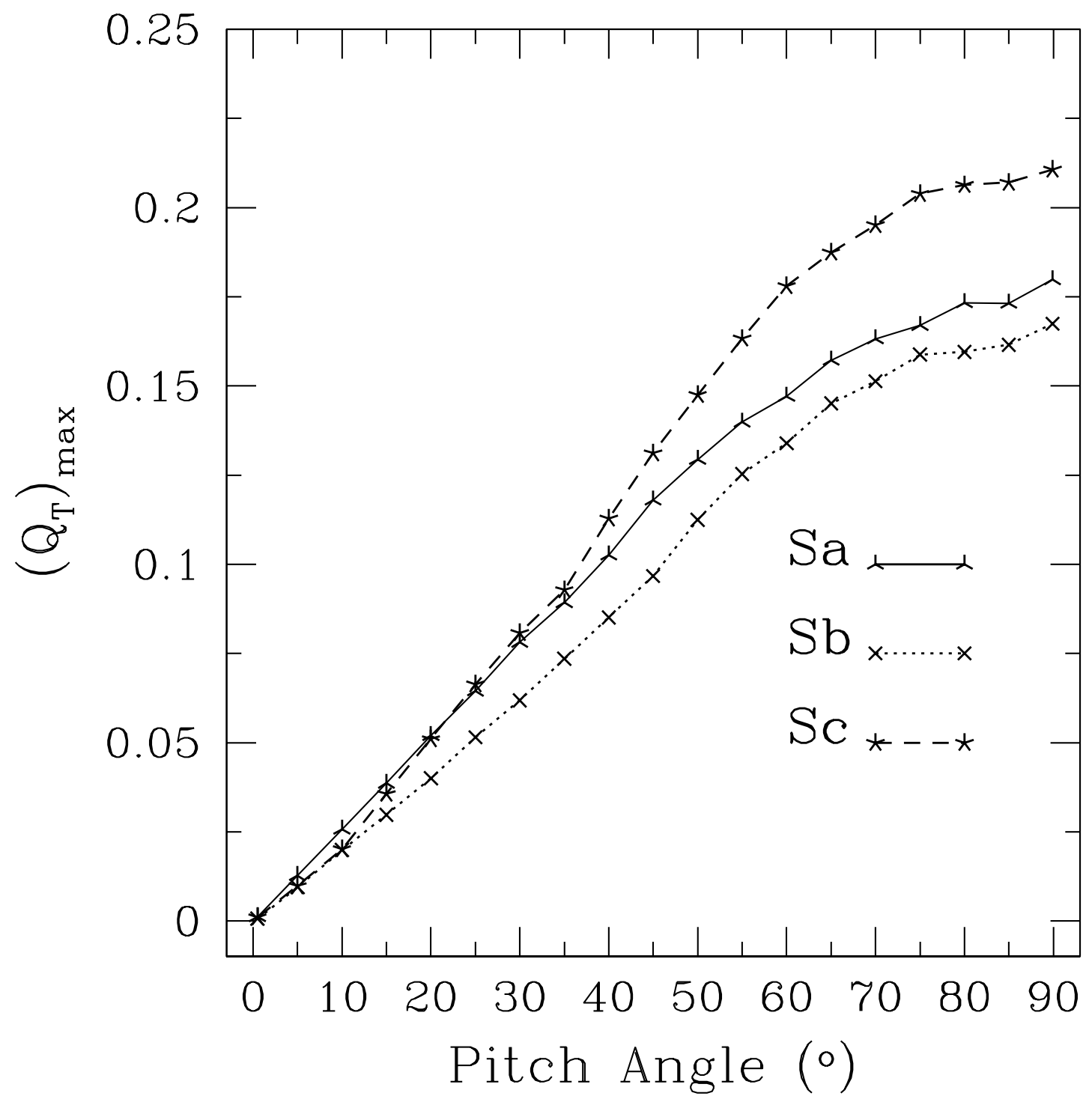

Fig. 4.- Maximum value, $\left(\mathrm{Q}_{\mathrm{T}}\right)_{\max }$, of the parameter $\mathrm{Q}_{T}(R)$ (maximum relative torques) vs. pitch angle of the spiral arms. Solid line gives $\left(\mathrm{Q}_{\mathrm{T}}\right)_{\max }$ for an Sa galaxy, dotted line for an Sb galaxy and dashed line for an Sc galaxy. 
Table 1. Parameters of the Galactic Models

\begin{tabular}{|c|c|c|c|c|}
\hline Parameter & \multicolumn{3}{|c|}{ Value } & Reference \\
\hline & \multicolumn{3}{|c|}{ Spiral Arms } & \\
\hline & $\mathrm{Sa}$ & $\mathrm{Sb}$ & $\mathrm{Sc}$ & \\
\hline locus & & Logarithmic & & 1,9 \\
\hline arms number & & 2 & & 2 \\
\hline pitch angle $\left(^{\circ}\right)$ & $4-40$ & $8-45$ & $10-60$ & 3,7 \\
\hline $\mathrm{M}_{\text {arms }} / \mathrm{M}_{\text {disk }}$ & & $3 \%$ & & \\
\hline scale-length (disk based) ( kpc) & 7 & 5 & 3 & 4,5 \\
\hline $\begin{array}{l}\text { pattern speed }\left(\mathrm{km} \mathrm{s}^{-1} \mathrm{kpc}^{-1}\right) \\
\text { (clockwise) }\end{array}$ & -30 & -25 & -20 & 1,6 \\
\hline ILR position ( $\mathrm{kpc}$ ) & 3.0 & 2.29 & 2.03 & \\
\hline corotation position $(\mathrm{CR})(\mathrm{kpc})$ & 10.6 & 11.14 & 8.63 & \\
\hline inner limit ( kpc) & 3.0 & 2.29 & 2.03 & $\begin{array}{l}\sim \text { ILR position } \\
\text { based }\end{array}$ \\
\hline \multirow[t]{2}{*}{ outer limit ( kpc) } & 10.6 & 11.14 & 8.63 & $\begin{array}{l}\sim \mathrm{CR} \text { position } \\
\text { based }\end{array}$ \\
\hline & \multicolumn{3}{|c|}{ Axisymmetric Components } & \\
\hline $\mathrm{M}_{\text {disk }} / \mathrm{M}_{\text {halo }}{ }^{1}$ & 0.07 & 0.09 & 0.1 & 4,8 \\
\hline $\mathrm{M}_{\text {bulge }} / \mathrm{M}_{\text {disk }}$ & 0.9 & 0.4 & 0.2 & 5,8 \\
\hline Rot. Curve $\left(\mathrm{V}_{\max }\right)\left(\mathrm{km} \mathrm{s}^{-1}\right)$ & 320 & 250 & 170 & 7 \\
\hline $\mathrm{M}_{\text {disk }}\left(\mathrm{M}_{\odot}\right)$ & $12.8 \times 10^{10}$ & $12.14 \times 10^{10}$ & $5.10 \times 10^{10}$ & 4 \\
\hline $\mathrm{M}_{\text {bulge }}\left(\mathrm{M}_{\odot}\right)$ & $11.6 \times 10^{10}$ & $4.45 \times 10^{10}$ & $1.02 \times 10^{10}$ & $\begin{array}{l}M_{\text {disk }} / M_{\text {bulge }} \\
\text { based }\end{array}$ \\
\hline $\mathrm{M}_{\text {halo }}\left(\mathrm{M}_{\odot}\right)$ & $1.64 \times 10^{12}$ & $1.25 \times 10^{12}$ & $4.85 \times 10^{11}$ & $\begin{array}{l}M_{\text {disk }} / M_{\text {halo }} \\
\text { based }\end{array}$ \\
\hline \multirow[t]{2}{*}{ Disk scale-length ( kpc) } & 7 & 5 & 3 & 4,5 \\
\hline & Constants o. & the Axisymmetric & Components $^{2}$ & \\
\hline Bulge $\left(\mathrm{M}_{\text {bulge }}, \mathrm{b}_{1}\right)^{3}$ & $5000,2.5$ & $2094.82,1.7$ & $440,1.0$ & \\
\hline Disk $\left(\mathrm{M}_{\text {disk }}, \mathrm{a}_{2}, \mathrm{~b}_{2}\right)^{3}$ & $5556.03,7.0,1.5$ & $5232.75,5.0,1.0$ & $2200,5.3178,0.25$ & \\
\hline Halo $\left(\mathrm{M}_{\text {halo }}, \mathrm{a}_{3}\right)^{3}$ & $15000,18.0$ & $10000,16.0$ & $2800,12.0$ & \\
\hline
\end{tabular}

${ }^{1} \mathrm{Up}$ to $100 \mathrm{kpc}$ halo radius.

${ }^{2}$ In galactic units, where a galactic mass unit $=2.32 \times 10^{7} \mathrm{M}_{\odot}$ and a galactic distance unit $=$ kpc. 
${ }^{3} \mathrm{~b}_{1}, \mathrm{a}_{2}, \mathrm{~b}_{2}$, and $\mathrm{a}_{3}$ are scale lengths.

References. - 1) Grosbol \& Patsis 1998. 2) Drimmel et al. 2000; Grosbøl et al. 2002. 3) Kennicutt 1981. 4) Pizagno et al. 2005 5) Weinzirl et al. 2009. 6) Patsis et al. 1991; Grosbøl \& Dottori 2009; Egusa et al. 2009; Fathi et al. 2009. 7) Brosche 1971; Ma et al. 2000; Sofue \& Rubin 2001. 8) Block et al. 2002. 9) Pichardo et al. 2003. 


\subsection{Orbital Analysis}

For the orbital analysis we employed periodic orbits and Poincaré diagrams. With this extensive phase space and configuration space orbital study, we are able to set two restrictions to one of the structural parameters of spiral arms: the pitch angle. The motion equations are solved in the non-inertial reference system of the spiral arms, and in Cartesian coordinates $\left(x^{\prime}, y^{\prime}, z^{\prime}\right)$.

\subsubsection{Periodic Orbits}

Periodic orbits represent the simplest orbits of potentials in general. They are also the most important orbits, because these are followed by sets of non-periodic orbits, and even for chaotic regions confined between two quasiperiodic orbits, librating around the periodic ones (i.e. forming tubes surrounding the periodic orbits). In self-consistent systems, periodic orbits support large-scale structures, such as bars and spiral arms, they are known as the "dynamic backbone" of potentials.

We computed between 40 and 60 periodic orbits for each Poincaré diagram, using the Newton-Raphson method. As a first guess for the initial conditions in the calculation of the periodic orbit the code provides the periodic orbit at a given radius in the background axisymmetric potential. The orbits are launched from $y^{\prime}=0$, on the $x^{\prime}$-axis $>0$ with $v_{x}^{\prime}=0$ and $v_{y}^{\prime}=v_{c}$, where $v_{c}$ is the corresponding circular velocity given by,

$$
v_{c}=\left(x^{\prime}\left|\frac{d \Phi_{0}}{d x^{\prime}}\right|\right)^{1 / 2},
$$

and $\Phi_{0}$ is the axisymmetric potential.

Periodic orbits close themselves in one or more periods; we use this property to find them. In this manner, the Newton-Raphson method searches a root based on the minimum distance and velocity between the final and initial points of the orbit after it completes a period. When the method finds a root, we obtain a periodic orbit.

\subsubsection{Density Response}

To calculate the density response to a spiral potential, we employ the method of Contopoulos \& Grosbøl (1986), that quantifies the support with periodic orbits to the arms. The 
method assumes that stars in circular orbits in an axisymmetric potential, rotating in the same direction of the spiral perturbation, will be trapped around the corresponding periodic orbit in the presence of the spiral arms. To this purpose, we computed a large set of periodic orbits and calculated the density response along their extension using the conservation of mass flux between any two successive orbits. With this information we search the position of the density response maxima along each periodic orbit. The locus of the obtained positions is compared with the imposed locus of PERLAS.

We calculated the average density response around each one of these response maxima, taking a circular vicinity with a radius of 500 pc. We then compared the density response with the imposed density. The imposed density is the sum of the axisymmetric disk density on the galactic plane and the central density of the spiral arms.

\subsubsection{Poincaré Diagrams}

In Poincaré diagrams ordered orbits appear as invariant one-dimensional curves; periodic orbits, on the other hand, draw a finite set of dots. In phase space diagrams, chaotic orbits appear as scattered sets of dots.

In the non-inertial frame, the effective potential on the galactic plane is given by:

$$
\Phi_{\mathrm{eff}}\left(x^{\prime}, y^{\prime}\right)=\Phi_{0}+\Phi_{s p}\left(x^{\prime}, y^{\prime}\right)-\frac{1}{2} \Omega_{p}^{2}\left(x^{2}+y^{\prime 2}\right)
$$

where $\Phi_{0}$ is the axisymmetric potential, $\Phi_{s p}$ is the potential of the spiral arms, and $\Omega_{p}$ is its angular velocity. The only known analytical integral of stellar motion in the non-inertial system of reference is the Jacobi constant, given by

$$
E_{J}=\frac{1}{2} v^{\prime 2}+\Phi_{\mathrm{eff}}
$$

where $v^{\prime}$ is the star velocity. Poincaré diagrams are constructed following the usual procedure. They present two regions, each one containing 50 orbits with 300 points each (corresponding to the number of periods), with a given prograde or retrograde sense of rotation, defined in the galactic non-inertial frame. In our models, the spiral patterns move in the clockwise sense. Therefore, the right side of the diagram (launching orbits with $x^{\prime}>0, v_{y}^{\prime}>0$ ) is the retrograde region, while the left side (with $x^{\prime}<0, v_{y}^{\prime}>0$ ), is the prograde region. 


\section{Results}

We carried out an extensive orbital study with periodic orbital analysis, density response and studies in the phase space to determine whether limit values to different structural parameters of normal spiral galaxies can be established.

We produced axisymmetric potential models to simulate typical $\mathrm{Sa}, \mathrm{Sb}$ and $\mathrm{Sc}$ spiral galaxies, and superposed a spiral arms potential (PERLAS) to study the stellar orbital behavior in the disk, as we changed the pitch angle for each galaxy type. Normal spiral galaxies present a wide scatter in the pitch angle, ranging from $\sim 4^{\circ}$ to $50^{\circ}$. We have found two restrictions for the pitch angle in normal spiral galaxies, the first based on ordered behavior and the second based on chaotic behavior.

\subsection{Pitch Angle Restriction Based on Ordered Motion: long lasting or transient spiral arms}

We estimated the self-consistency of each one of our models through the construction of periodic orbits. The existence of periodic orbits makes it more likely in steady conservative potentials, the support to long-lasting large-scale structures. We present a periodic orbital study for each morphological type (see Figures 5-77). In addition, we search the position of the density response maxima along each periodic orbit. We compared these positions with the center of the imposed spiral arms. Figures 5- 7 show the response maxima as filled squares, where the orbits crowd producing a density enhancement, and the imposed spiral pattern are represented by open squares. In these figures we present periodic orbits for Sa, $\mathrm{Sb}$ and Sc galaxies (Figure 5, 6, and 7, respectively). We have used the same axisymmetric background potential for each morphological type, based on the parameters presented in Table 1. The pitch angle in these figures ranging from $4^{\circ}$ to $40^{\circ}$ for an Sa galaxy, from $8^{\circ}$ to $45^{\circ}$ for an $\mathrm{Sb}$ galaxy and from $10^{\circ}$ to $60^{\circ}$ for an Sc galaxy.

In Figure 5, we see how for smaller pitch angles $\left(i \lesssim 15^{\circ}\right)$, the density response maxima coincides with the imposed spiral arms potential. This means that the filled squares in the figure, that represent the places in the arm where stars would crowd for long times, settle down along the locus of the imposed spiral, making the existence of stable long-lasting spiral arms more likely. On the other hand, for spiral arms with pitch angles larger than $\sim 15^{\circ}$, the response maxima lag behind systematically the imposed spiral arm potential, i. e., the pitch angles corresponding to the density response are smaller than the imposed ones.

Figure 6 shows a similar behavior than the one for Sa galaxies, but in this case, the pitch angle range is slightly wider. For smaller pitch angles $\left(i \lesssim 18^{\circ}\right)$ the density response 
supports closely the imposed spiral arms. For $i>18^{\circ}$, the density response produces spiral arms with smaller pitch angles than the imposed locus, avoiding the support to the spiral structure.

Figure 7 shows a similar behavior than $\mathrm{Sa}$ and $\mathrm{Sb}$ galaxies, but in this case, the pitch angle range is wider. For smaller pitch angles $\left(i \lesssim 20^{\circ}\right)$ the density response seems to support the imposed spiral arms. For $i>20^{\circ}$, the density response produces spiral arms with smaller pitch angles than the imposed locus, avoiding to support the spiral structure.

In the three types of galaxies, with pitch angles beyond $15^{\circ}, 18^{\circ}$ and $20^{\circ}$ for $\mathrm{Sa}, \mathrm{Sb}$, and Sc galaxies, respectively, the density response seems to "avoid" open long-lasting spiral arms. Long-lasting spiral arms are not supported anymore after these limits; spiral arms in these cases may be rather transient structures. In Figure 8 we show a plot of phase angular difference between the imposed spiral potential and the density response $v s$. pitch angle of the imposed spiral arms.

An additional method to complement and to reinforce the results given by periodic orbits, is the comparison of the spiral arms density response (filled squares in Figures 9 11) with the imposed density (open squares in Figures 9 - 11). In Figure 9, we present the densities (the spiral arms density response, and the spiral arms imposed density, i.e. PERLAS), for an Sa galaxy. As the density response maxima in the previous diagrams, this figure shows that for pitch angles up to $\sim 15^{\circ}$, the density response fits well with the imposed density. In Figure 10, we present the densities for an Sb galaxy. In this figure we see almost the same behavior than in Figure 9, but in this case, the pitch angle limit, where the density response does not fit the imposed arm, is $\sim 18^{\circ}$. Finally, in Figure 11, we present the same diagrams for an Sc galaxy. Here, the behavior is very similar to the Sa and Sb cases, but the limit for the pitch angle, where the density response is fitted to the imposed density, is $\sim 20^{\circ}$.

With periodic orbits, maxima density response, and comparing the density response and imposed density, in order to obtain support to long-lasting spiral arms, the pitch angle should be smaller than $\sim 15^{\circ}, 18^{\circ}$ and $20^{\circ}$, for Sa, Sb and Sc galaxies, respectively. However,

spiral arms evidently exist with larger pitch angles in galaxies, we propose then that these are rather in a transient form.

\subsection{Pitch Angle Restriction Based on Chaotic Motion}

Confined chaotic orbits are able to support large-scale structures, such as spiral arms (Patsis \& Kalapotharakos 2011; Kaufmann \& Contopoulos 1996; Contopoulos \& Grosbøl 
1986). However, grand design structures are not expected to arise from systems where chaos fully dominates (Voglis et al. 2006). In this study we found a restriction based on chaotic behavior. To do this, we have produced an extensive study of the Jacobi energy families in the phase space, as a function of the pitch angle in normal spiral galaxies. In this section we show that there is a limit to the pitch angle, for which chaos becomes pervasive destroying all periodic orbits and the ordered orbits surrounding them, in the relevant spiral arm region.

We present here a set of phase space diagrams for each morphological type (Figures 12 - 14). As in the study of periodic orbits (Section 3.1), we employed an axisymmetric background potential for an Sa, Sb and Sc galaxy, based on the parameters given in Table1. In these experiments we have only varied the pitch angles. Each mosaic has 20 panels, that show phase space diagrams with different Jacobi constant families ranging from $E_{J}=-4050$ to $-3278 \times 10^{2} \mathrm{~km}^{2} \mathrm{~s}^{-2}$ for an Sa galaxy (where $E_{J}=-4100,-3410,-3275 \times 10^{2} \mathrm{~km}^{2} \mathrm{~s}^{-2}$ correspond approximately to the positions of ILR, $4 / 1$ and CR resonances, respectively); from $E_{J}=-3150$ to $-2445 \times 10^{2} \mathrm{~km}^{2} \mathrm{~s}^{-2}$ for an Sb galaxy (where $E_{J}=-3290,-2550,-2442$ $\times 10^{2} \mathrm{~km}^{2} \mathrm{~s}^{-2}$ correspond approximately to the positions of ILR, 4/1 and CR resonances, respectively), and from $E_{J}=-1080$ to $-1021 \times 10^{2} \mathrm{~km}^{2} \mathrm{~s}^{-2}$ for an Sc galaxy (where $E_{J}=$ $-1280,-1075,-1022 \times 10^{2} \mathrm{~km}^{2} \mathrm{~s}^{-2}$ correspond approximately to the positions of ILR, 4/1 and CR resonances, respectively), covering the total extension of spiral arms.

Figure 12 shows Poincaré diagrams for an Sa galaxy, going from $4^{\circ}$ (top line of diagrams) to $30^{\circ}$ (bottom line of diagrams). For pitch angles between $4^{\circ}$ and $10^{\circ}$ (first two lines of diagrams), the ordered orbits are dominating and simple, periodic orbits support spiral arms up to corotation, approximately. The onset of chaos is clear at about $10^{\circ}$. As a function of the pitch angle, for $19^{\circ}$ (third line of diagrams), the orbital behavior is more complex, and it presents resonant islands. For $30^{\circ}$ (bottom line of diagrams), the chaotic region covers most of the regular prograde orbits. For pitch angles beyond $\sim 30^{\circ}$, chaos destroys periodic orbits.

Figure 13 shows Poincaré diagrams for an Sb galaxy, going from $12^{\circ}$ (top line of diagrams) to $40^{\circ}$ (bottom line of diagrams). For pitch angles of $10^{\circ}$ or less, the ordered orbits dominate. As we increase the pitch angles, at $12^{\circ}$ (first line of diagrams), the orbital behavior is mainly ordered, but close to corotation there is already a small chaotic region. For $21^{\circ}$ (second line of diagrams), the onset of chaos is clear, and there is a variety of complicate orbits. For $30^{\circ}$ (third line of diagrams), the chaotic region increases, and the orbital structure becomes much more complex. For $40^{\circ}$ (bottom line of diagrams), the chaotic region covers almost all regular prograde orbits. For pitch angles beyond $\sim 40^{\circ}$ chaos destroys periodic orbits.

In Figure 14, we present Poincaré diagrams for an Sc galaxy, going from $20^{\circ}$ (top line of 
diagrams) to $50^{\circ}$ (bottom line of diagrams). For a pitch angle of $20^{\circ}$ (first line of diagrams) or smaller, orbits are always ordered and quite simple, the spiral arms are supported up to corotation. For a pitch angle of $30^{\circ}$, the orbital behavior is slightly more complex, it shows some resonant islands and the chaotic region becomes already important, but this is still contained by stable periodic orbits. For $40^{\circ}$, chaos dominates the region around the stable periodic orbits. For $50^{\circ}$ (bottom line of diagrams), chaos covers practically all the regular prograde region, very close to the main periodic orbits. For pitch angles larger than $\sim 50^{\circ}$ periodic orbits are destroyed by fully developed chaos. It might be natural wondering why for bars it is "permitted" to have a $90^{\circ}$ pitch angle, although we are stating here it is not possible for spiral arms. However, comparing directly the bar and the spiral arms regions is not straightforward, since the density structure and dynamics of the regions where they belong are quite different.

The onset of chaos begins in the prograde region of Poincaré diagrams. The main cause for chaotic motion has been attributed to resonance interactions (Contopoulos 1967; Martinet 1974; Athanassoula et al. 1983; Pichardo et al. 2003). In the case of retrograde orbital regions, resonances are more widely separated than in the progade resonances case, which may explain why the onset and well developed chaos takes place in the prograde region first (and in general, only). However, it is worth noticing that in our experiments, we have mantained the same pattern angular speeds, that places resonances, at the same radii, increasing only pitch angles, and we have noticed that chaos (not originated only by the resonances position), increases dramatically. This means that at a given position of the resonances (posed by the angular speeds), increasing pitch angles, will widen the chaotic regions.

\section{Discussion and Conclusions}

We have produced a set of models for observationally motivated potentials to simulate typical Sa, Sb and Sc spiral galaxies with three dimensional bisymmetric spiral arms. Observed galaxies, classified as $\mathrm{Sa}$, Sb or Sc, present a wide scatter in pitch angles going from $\sim 4^{\circ}$ to $25^{\circ}$ for Sa galaxies, from $\sim 8^{\circ}$ to $35^{\circ}$ for $\mathrm{Sb}$ galaxies and from $\sim 10^{\circ}$ to $50^{\circ}$ for $\mathrm{Sc}$ galaxies. With our models, we studied extensively the stellar dynamical effects of the spiral arms pitch angle on the plane of the disk, ranging from $4^{\circ}$ to $40^{\circ}$, for an Sa galaxy, from $8^{\circ}$ to $45^{\circ}$, for an $\mathrm{Sb}$ galaxy and from $10^{\circ}$ to $60^{\circ}$, for an Sc galaxy.

We found two important restrictions to the pitch angle. The first restriction is based on the orbital ordered behavior. With the study of periodic orbits and density response, we found that there is an abrupt limit for the density response at approximately $15^{\circ}$ for 
Sa galaxies, $18^{\circ}$ for $\mathrm{Sb}$ galaxies and $20^{\circ}$ for $\mathrm{Sc}$ galaxies. This limit denotes the end of orbital support of the density response to the imposed spiral arm potential. In cases, where the spiral arms potential is followed by their density response produced by periodic orbital crowding, the spiral arms are more stable and could be explained better as a long-lasting feature. Beyond these limits, the density response produces systematically smaller pitch angles than the imposed spiral arms. Galaxies with spiral arms beyond these limits would rather be explained as transient features.

We found a second restriction, this time based on chaotic orbital behavior. In this case, we find a limit for the very same existence of spiral arms for each morphological type: for an Sa, the limit is $\sim 30^{\circ}$, for an Sb, the limit is $\sim 40^{\circ}$, and for an Sc the limit is $\sim 50^{\circ}$, for which spiral arms are so open that chaos dominates large regions of phase space, but there exist periodic orbits supporting spiral arms. Beyond these limits for each type of galaxy, chaos becomes pervasive destroying all orbital support.

With this orbital study, we are able to pose both a limit for steady long-lasting spiral arms, beyond which spiral arms are better explained as transient features and a limit for maximum pitch angles (no matter their nature) in normal spiral galaxies before the system becomes completely chaotic.

Several structural and dynamical parameters may play an important role in the stellar dynamics behavior, such as the pattern speed, the strength of the spiral arms (spiral arms mass), the density decay along the spiral arms, the axisymmetric components mass ratios, etc. In this paper we have isolated the effect of the pitch angle on stellar dynamics to understand at what extent we could impose restrictions to its properties in models for spiral galaxies based on order and chaos. We employ for this purpose, realistic values for the rest of the parameters that identify approximately typical Sa, Sb and Sc galaxies. A comprehensive study for other parameters is being prepared in an forthcoming paper. We provide here preliminary results on those studies for three of the relevant parameters within the observational or theoretical values for spiral galaxies: pattern speed, spiral arms mass and density decay of the spiral arms. For the pattern speed, although the construction and radial extension of periodic orbits (length of the spiral arms) depends sensibly on the pattern speed, the existence of the periodic orbits supporting the spiral structure inside the corotation resonance (or 4/1 resonance) seems not too sensitive, within the observed (20 to $30 \mathrm{~km} \mathrm{~s}^{-1} \mathrm{kpc}^{-1}$ ) limits for real spiral galaxies. This means that, within the observed limits for spiral angular speeds in galaxies, the effect on the restriction of the angular speed on whether spiral arms are transient or long lived structures seems to be much less important. The spiral to disk mass ratio, as well as the angular speed, is a very restricted quantity by observations and theory. Translating the amplitudes or relative forcing, to spiral arms mass, 
the masses to obtain self-consistent models go upto 5 or $6 \%$ of the mass of the disk. As long as we change within these limits the spiral arms mass, the restriction to the pitch angle for

the spiral arms to be supported by periodic orbits is only slightly sensitive. Regarding the density fall of the spiral arms we studied several types of both lineal decay and exponential decay. This specific parameter results of little consequence as long as the linear decay slope goes approximately similar to the average slope of the exponential fall in the first half of the spiral arms extent. In a future work, we will present a detailed stellar dynamical study with these and other parameters of spiral galaxies.

We acknowledge the anonymous referee for enlightening comments that improved this work. We thank DGAPA-PAPIIT through grants IN110711 and IN112911.

\section{REFERENCES}

Allen, C. \& Santillán, A. 1991, Rev. Mexicana Astron. Astrofis., 22, 256

Antoja, T., Valenzuela, O., Pichardo, B., Moreno, E., Figueras, F. \& Fernández D. 2009, ApJ, 700, L78

Antoja, T., Figueras, F., Romero-Gómez, M., et al. 2011, MNRAS, 418, 1423

Athanassoula, E., Bienaymé, O., Martinet, L., \& Pfenniger, D. 1983, A\&A, 127, 349

Block, D. L., Bournaud, F., Combes, F., Puerari, I. \& Buta, R. 2002,A\&A, 394, L35

Block, D. L., Buta, R., Knapen, J. H., et al. 2004, AJ, 128, 183

Binney, J. \& Tremaine, S. 1994, Galactic Dynamics(Pinceton University Press)

Brosche, P. 1971, A\&A, 13, 293

Buta, R. \& Block, D. L. 2001, ApJ, 550, 243

Buta, R., Laurikainen, E., \& Salo, H. 2004, AJ, 127, 279

Buta, R., Vasylev, S., Salo, H. \& Laurikainen, E. 2005, AJ, 130, 523

Combes, F. \& Sanders, R. H. 1981, A\&A, 96, 164

Contopoulos, G. 1967, Bull. Astron. (Ser. 3), 2, 223

Contopoulos, G. 1983, A\&A, 117, 89 
Contopoulos, G. 1995, NYASA, 751, 112

Contopoulos, G. 2002, Order and Chaos in Dynamical Astronomy, Springer, NY

Contopoulos, G. \& Grosbøl, P. 1986, A\&A, 155, 11

Contopoulos, G., \& Grosbol, P. 1988, A\&A, 197, 83

Contopoulos, G. \& Patsis, P. A., eds., 2008, Chaos in Astronomy, Springer, Berlin

Contopoulos, G., Varvoglis, H. \& Barbanis, B. 1987, A\&A, 172, 55

Danver, C. G. 1942, Ann. Lund. Obs., 10, 162

Davis, B. L., Berrier, J. C., Shields, D. W., et al. 2012, ApJS, 199, 33

de Vaucouleurs G. 1959, Hanbuch der Physik, 53, 275

Drimmel, R. 2000, A\&A, 358, L13

Egusa, F., Kohno, K., Sofue, Y., Nakanishi, H., \& Komugi, S. 2009, ApJ, 697, 1870

Fathi, K., Beckman, J. E., Piñol-Ferrer, N., Hernández, O., Martínez-Valpuesta, I. \& Carignan, C. 2009, ApJ, 704, 1657

Foyle, K., Rix, H.-W., Dobbs, C. L., Leroy, A. K., \& Walter, F. 2011, ApJ, 735, 101

Fujii, M. S., \& Baba, J. 2012, MNRAS, 427, L16

Goldreich, P., \& Lynden-Bell, D. 1965, MNRAS, 130, 125

Groot, H. 1925, MNRAS, 85, 535

Grosbøl, P. 2003, LNP, 626, 201

Grosbøl, P., \& Dottori, H. 2009, A\&A, 499, L21

Grosbol, P. J. \& Patsis, P. A. 1998, A\&A, 336, 840

Grosbøl, P., Pompei, E., \& Patsis, P. A. 2002, Disks of Galaxies: Kinematics, Dynamics and Peturbations, 275, 305

Holmberg, E. 1958, Medd. Lund. Ast. Obs. Ser. II, No. 136

Hubble, E. P. 1926, ApJ, 64,321

Hubble, E. P. 1936, The Realm of the Nebulae. New Haven: Yale Univ. Press 
Julian, W. H., \& Toomre, A. 1966, ApJ, 146, 810

Kalapotharakos, C., Patsis, P. A. \& Grosbøl, P. 2010, MNRAS, 403, 83

Kaufmann, D. E., \& Contopoulos, G. 1996, A\&A, 309, 381

Kawata, D., Grand, R. J. J., \& Cropper, M. 2012, European Physical Journal Web of Conferences, 19, 7006

Kennicutt, R. 1981, AJ, 86, 1847

Laurikainen, E., Salo, H., Buta, R., \& Vasylyev, S. 2004, MNRAS, 355, 1251

Laurikainen, Eija \& Salo, Heikki 2002, MNRAS, 337, 1118

Lin, C. C. \& Shu, F. H. 1964, Apj, 140, 646

Ma, Jhun, Zhao, Jun-liang, Zhang, Fei-peng, \& Peng, Qiu-he 2000, Chinese Astronomy and Astrophysics, 24, 435

Ma, J. 2002, A\&A, 388, 389

Martinet, L. 1974, A\&A, 32, 329

Martos, M., Hernandez, X., Yáñez, M., Moreno, E. \& Pichardo, B. 2004, MNRAS, 350, 47

Maupomé, L., Pişmiş, P. \& Aguilar, L. 1981, Rev. Mexicana Astron. Astrof., 6, 45

Miyamoyo, M., \& Nagai, R. 1975, Pub. Astr. Soc. Japan, 27, 533

Patsis, P. A. 2008, AN, 329, 930

Patsis, P. A., Contopoulos, G. \& Grosbøl P. 1991, A\&A, 243, 373

Patsis, P. A., \& Kalapotharakos, C. 2011, Memorie della Societa Astronomica Italiana Supplementi, 18, 83

Patsis, P. A., Kaufmann, D. E., Gottesman, S. T. \& Boonyasait, V. 2009, MNRAS, 394, 142

Pérez-Villegas, A., Pichardo, B., Moreno, E., Peimbert, A., \& Velázquez, H. M. 2012, ApJL, $745, \mathrm{~L} 14$

Pichardo, B., Martos, M., Moreno, E. \& Espresate, J. 2003, ApJ, 582, 230

Pişmiş, P. \& Maupomé, L. 1978, Rev. Mexicana Astron. Astrof., 2, 319 
Pizagno, J., Prada, F., Weinberg, D. H., Rix, H. W., Harbeck, D., Grebel, E. K., Bell, E. F., Brinkmann, J., Holtzman, J. \& West, A. 2005, ApJ, 633, 844

Roberts, M. S. \& Haynes, M. P. 1994, ARA\&A, 32, 115

Roberts, W.W., Jr., Huntley, J. M., \& van Albada, G. D. 1979, ApJ, 233, 67

Rubin, V. C., Burstein, D., Ford, W. K., Jr., \& Thonnard, N. 1985, ApJ, 289, 81

Sage, L. J. 1993, A\&A, 272, 123

Sandage A. 1961, The Hubble Atlas of Galaxies. Carnegie Institution of Washington, Washington D.C.

Sandage A. 1975, Galaxies and Universe-Stars and Stellar Systems Chicago: University of Chicago Press

Sandage, A. 2000, PASP, 112, 504

Savchenko, S. S., \& Reshetnikov, V. P. 2011, Astronomy Letters, 37, 817

Seigar, M. S., Kennefick, D., Kennefick, J., \& Lacy, C. H. S. 2008, ApJ, 678, L93

Sellwood, J. A. 2011, MNRAS, 410, 1637

Sellwood, J. A., \& Carlberg, R. G. 1984, ApJ, 282, 61

Sersic, J. L. 1987, Extragalactic Astronomy, trans. Li Zong-yun, rev. Huang Ke-liang. Beijing: Science Press

Shields, D. W., Hughes, J. A., Barrows, S. R., et al. 2010, American Institute of Physics Conference Series, 1294, 283

Sofue, Y. \& Rubin, V. 2001, ARA\&A, 39, 137

Voglis, N., Stavropoulos, I. \& Kalapotharakos, C. 2006, MNRAS, 372, 901

Von der Pahlen 1911, AN, 188, 249

Vorobyov, E. I. 2006, MNRAS, 370, 1046

Weinzirl, T., Jogee, S., Khochfar, S., Burket, A. \& Kormendy, J. 2009, ApJ, 696, 411 


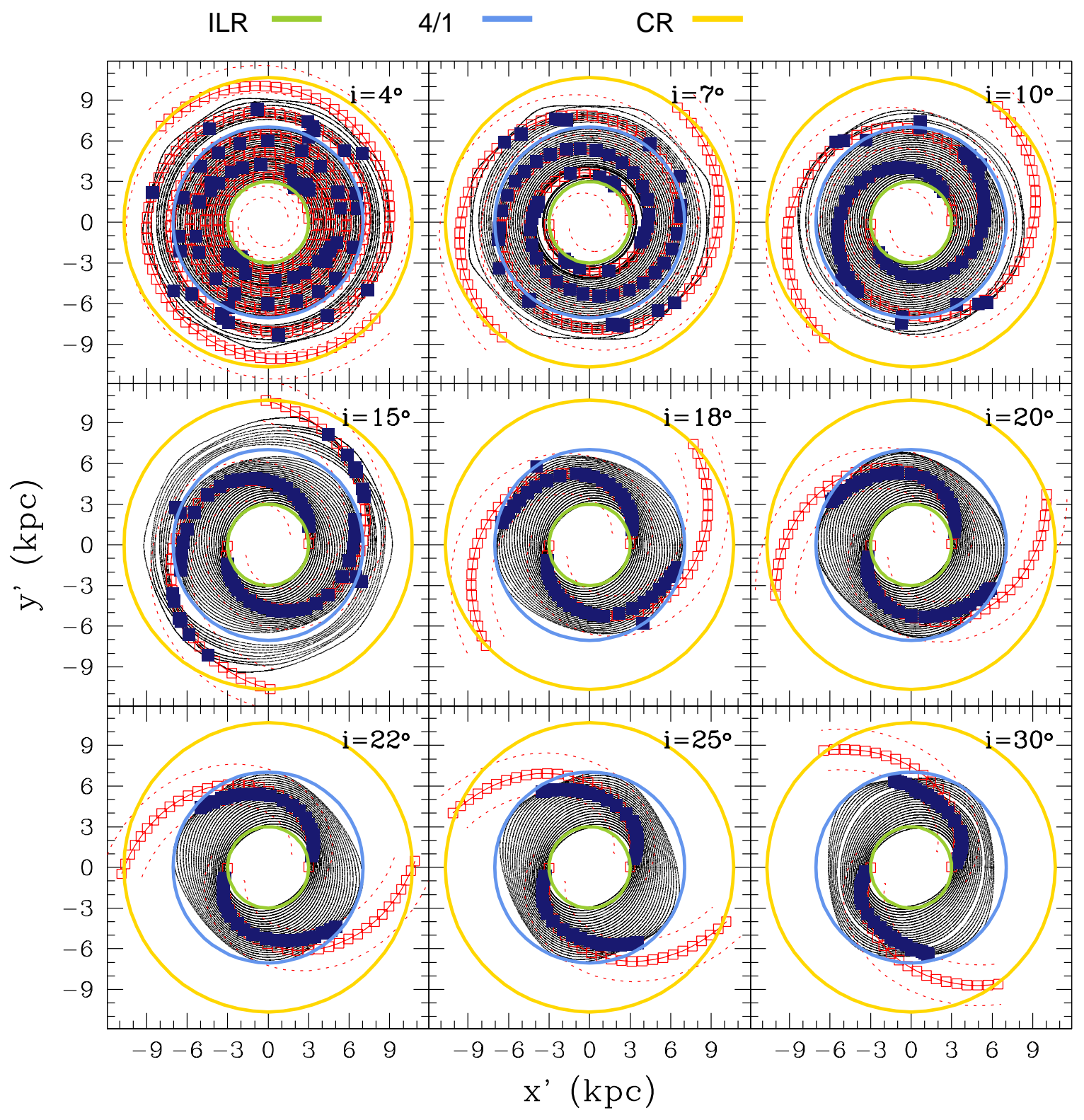

Fig. 5.- We present 9 panels showing the periodic orbits, response maxima (filled squares), and the spiral locus (open squares), for the three-dimensional spiral model of an Sa galaxy (Table 1), with pitch angles ranging from $4^{\circ}$ to $30^{\circ}$. 


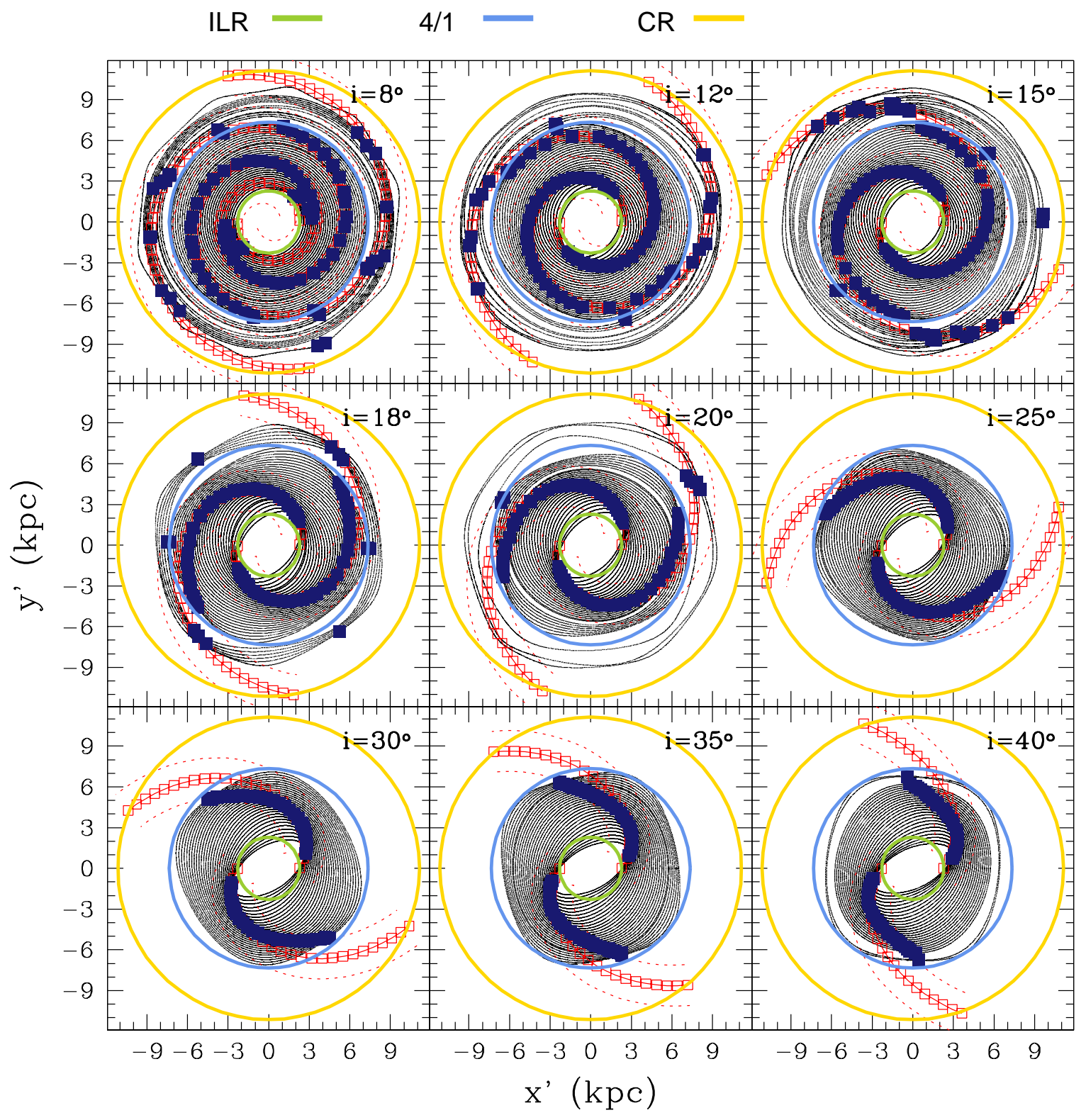

Fig. 6.-We present 9 panels showing the periodic orbits, response maxima (filled squares), and the spiral locus (open squares), for the three-dimensional spiral model of an Sb galaxy (Table 1), with pitch angles ranging from $8^{\circ}$ to $40^{\circ}$. 


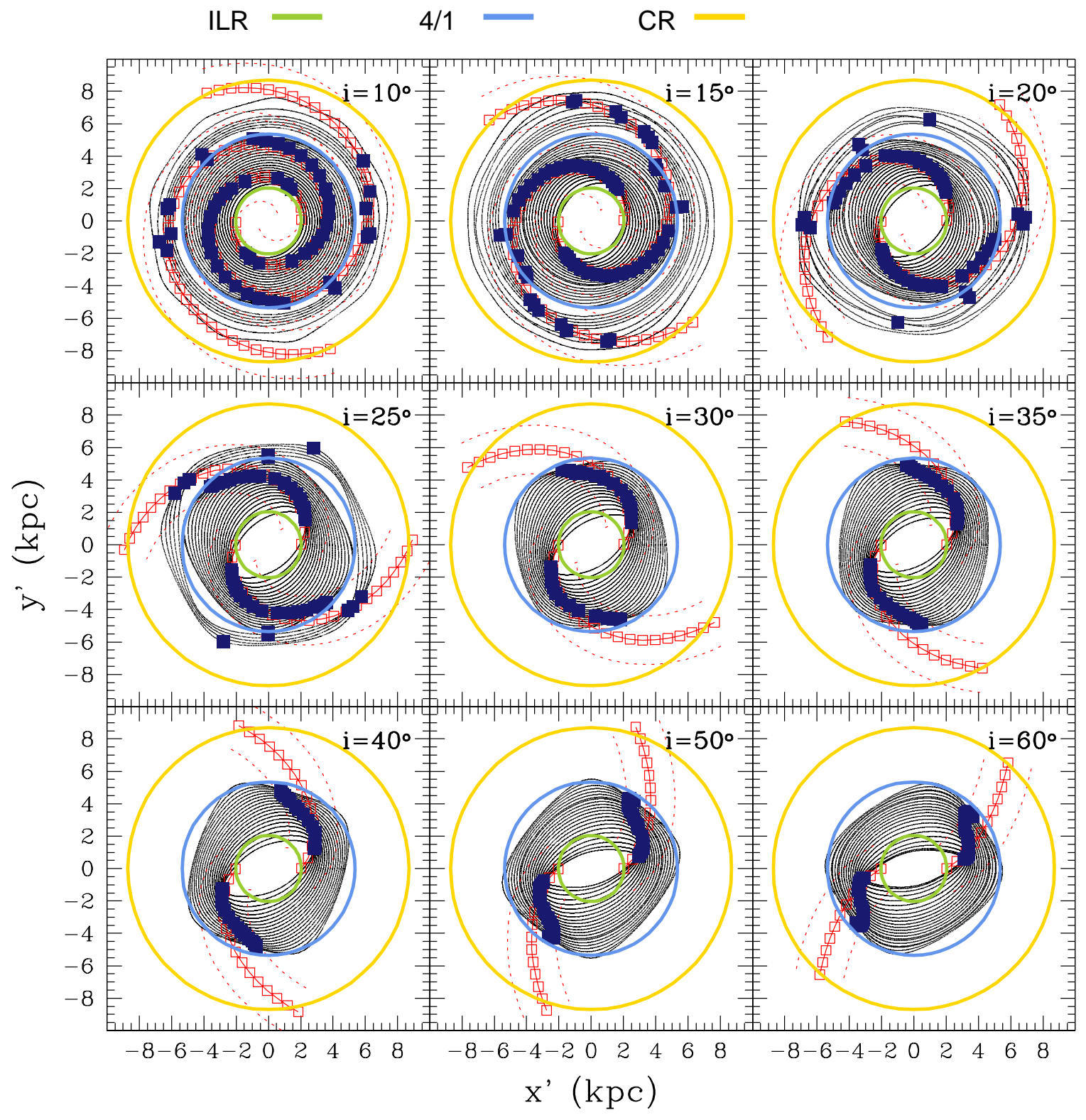

Fig. 7.- We present 9 panels showing the periodic orbits, response maxima (filled squares), and the spiral locus (open squares), for the three-dimensional spiral model of an Sc galaxy (Table 1), with pitch angles ranging from $10^{\circ}$ to $60^{\circ}$. 


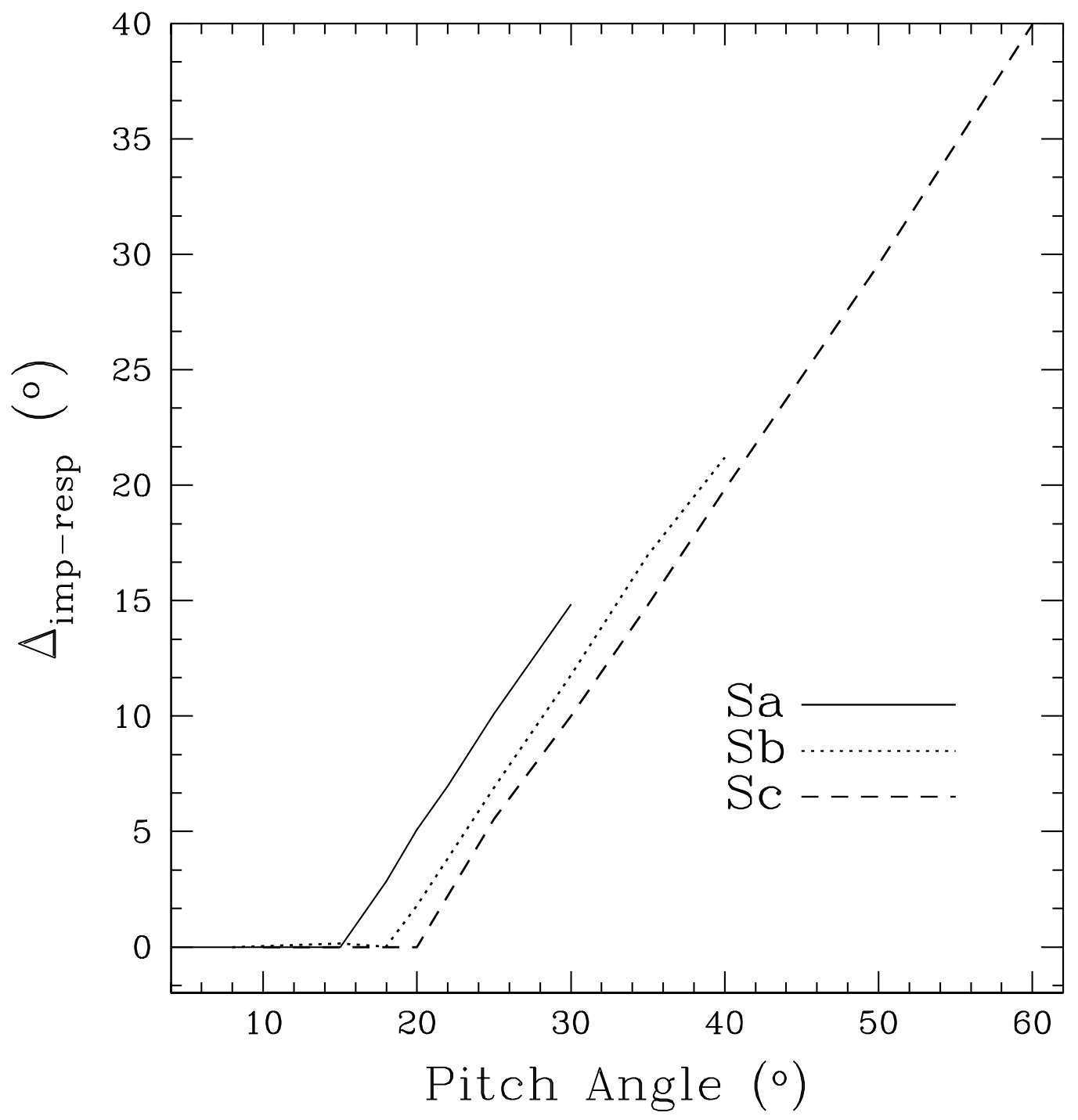

Fig. 8. - $\Delta_{\text {imp-resp }}$ is the phase angular difference between the density response and the imposed spiral potential. The solid line shows $\Delta_{i m p-r e s p}$ for an Sa galaxy, the dotted line corresponds an Sb galaxy and the dashed line to an Sc galaxy. 


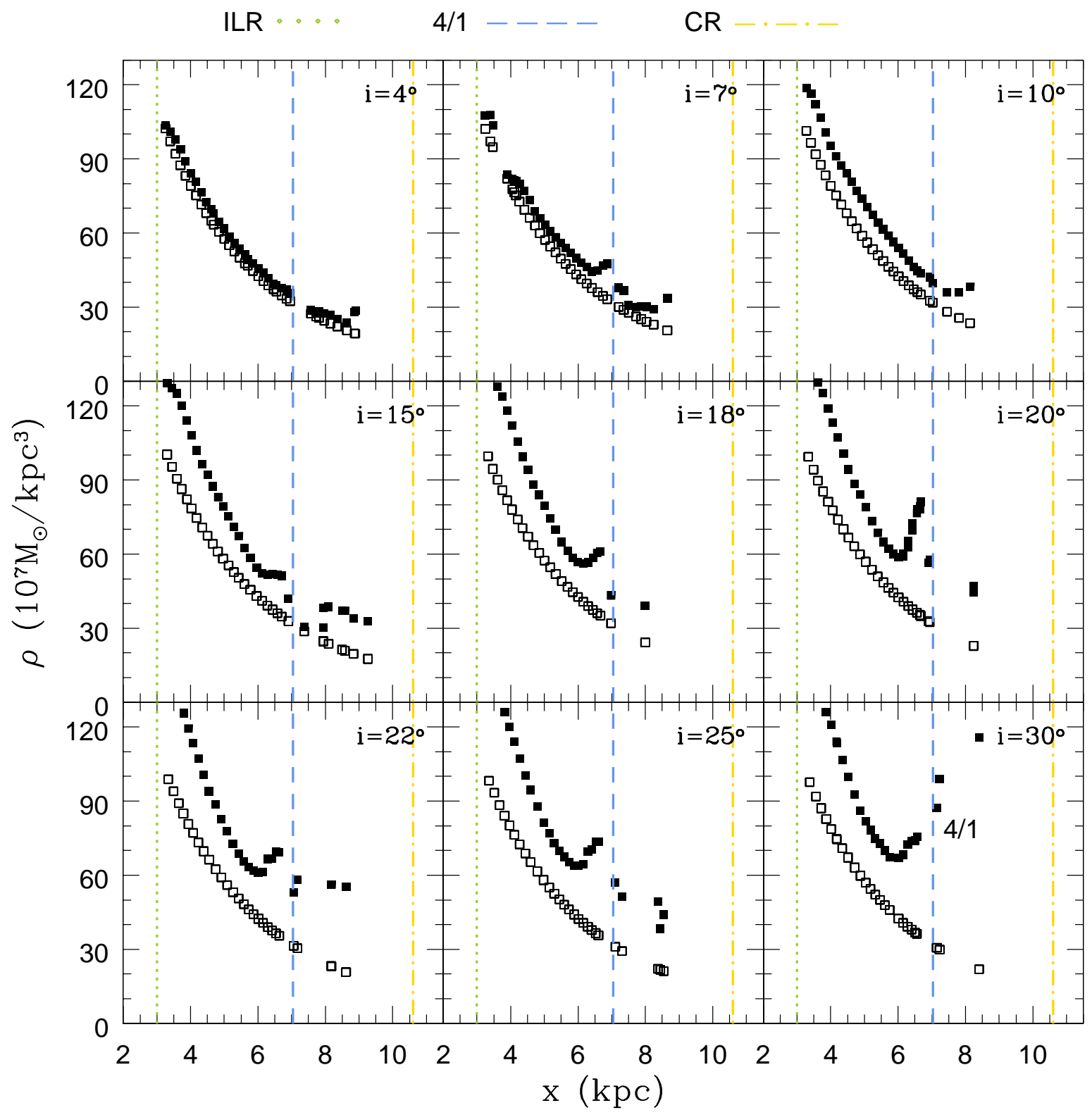

Fig. 9.- In this figure, filled squares represent the density response of the spiral arms for an Sa galaxy, and open squares represent the imposed density, with pitch angles ranging from $4^{\circ}$ to $30^{\circ}$. The dotted, dashed and dotted-dashed lines show the inner Linblad resonance (ILR) position, 4/1 resonance position and corotation resonance (CR) position, respectively. 


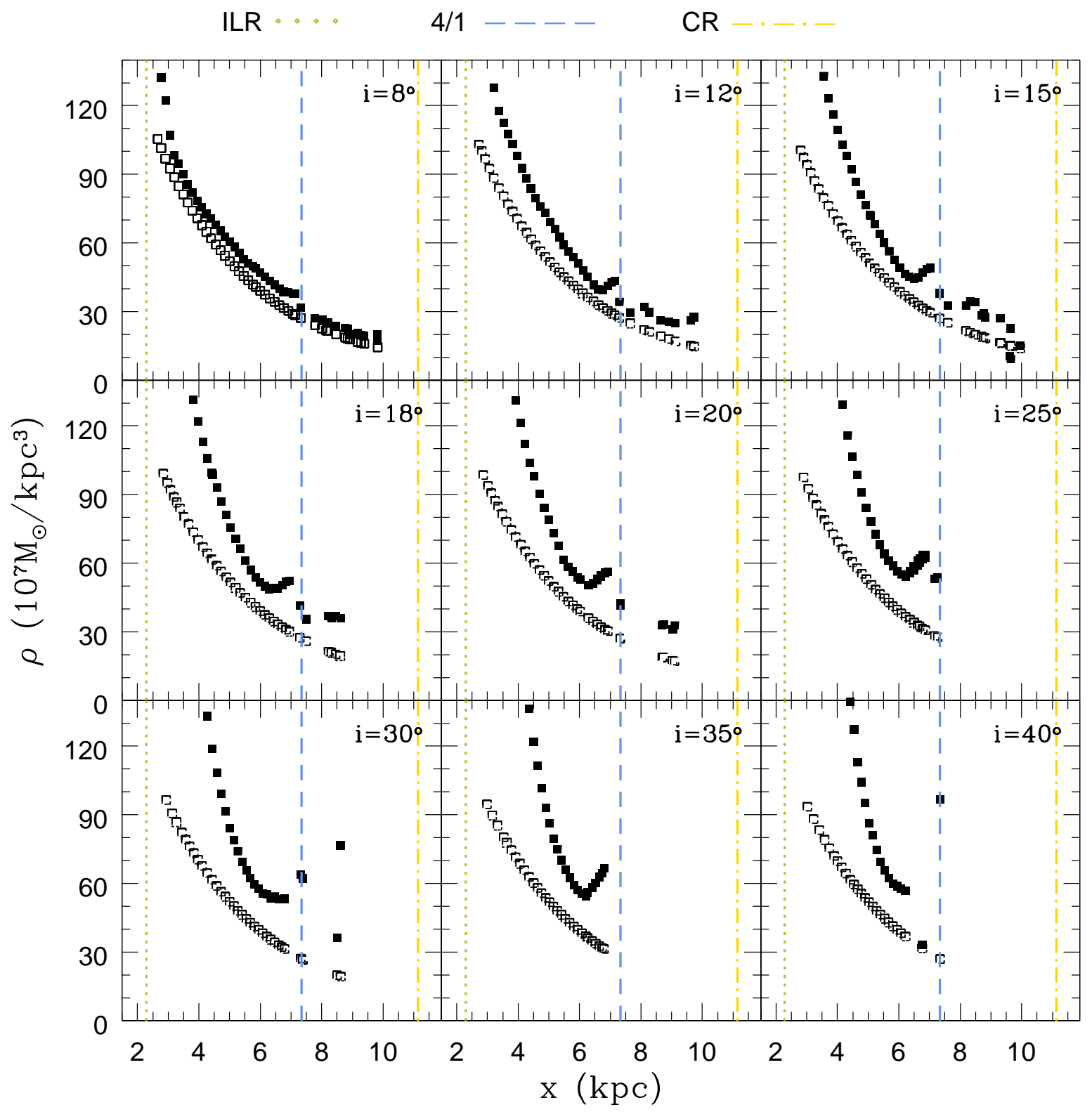

Fig. 10.- In this figure, filled squares represent the density response of the spiral arms for an Sb galaxy, and open squares represent the imposed density, with pitch angles ranging from $8^{\circ}$ to $40^{\circ}$. The dotted, dashed and dotted-dashed lines show the inner Linblad resonance (ILR) position, 4/1 resonance position and corotation resonance (CR) position, respectively. 


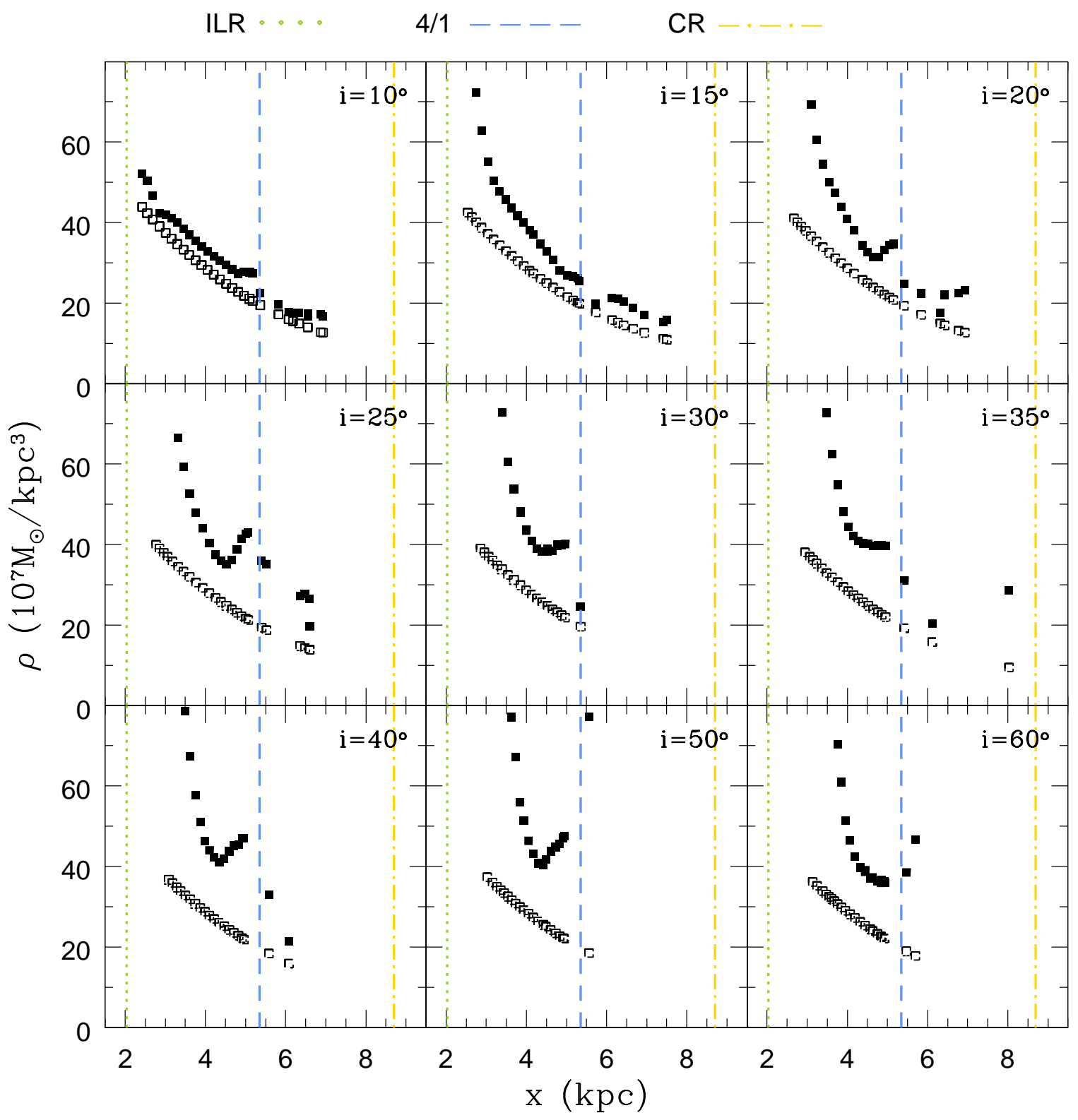

Fig. 11. - In this figure, filled squares represent the density response of the spiral arms for an Sc galaxy, and open squares represent the imposed density, with pitch angles ranging from $10^{\circ}$ to $60^{\circ}$. The dotted, dashed and dotted-dashed lines show the inner Linblad resonance (ILR) position, 4/1 resonance position and corotation resonance (CR) position, respectively. 


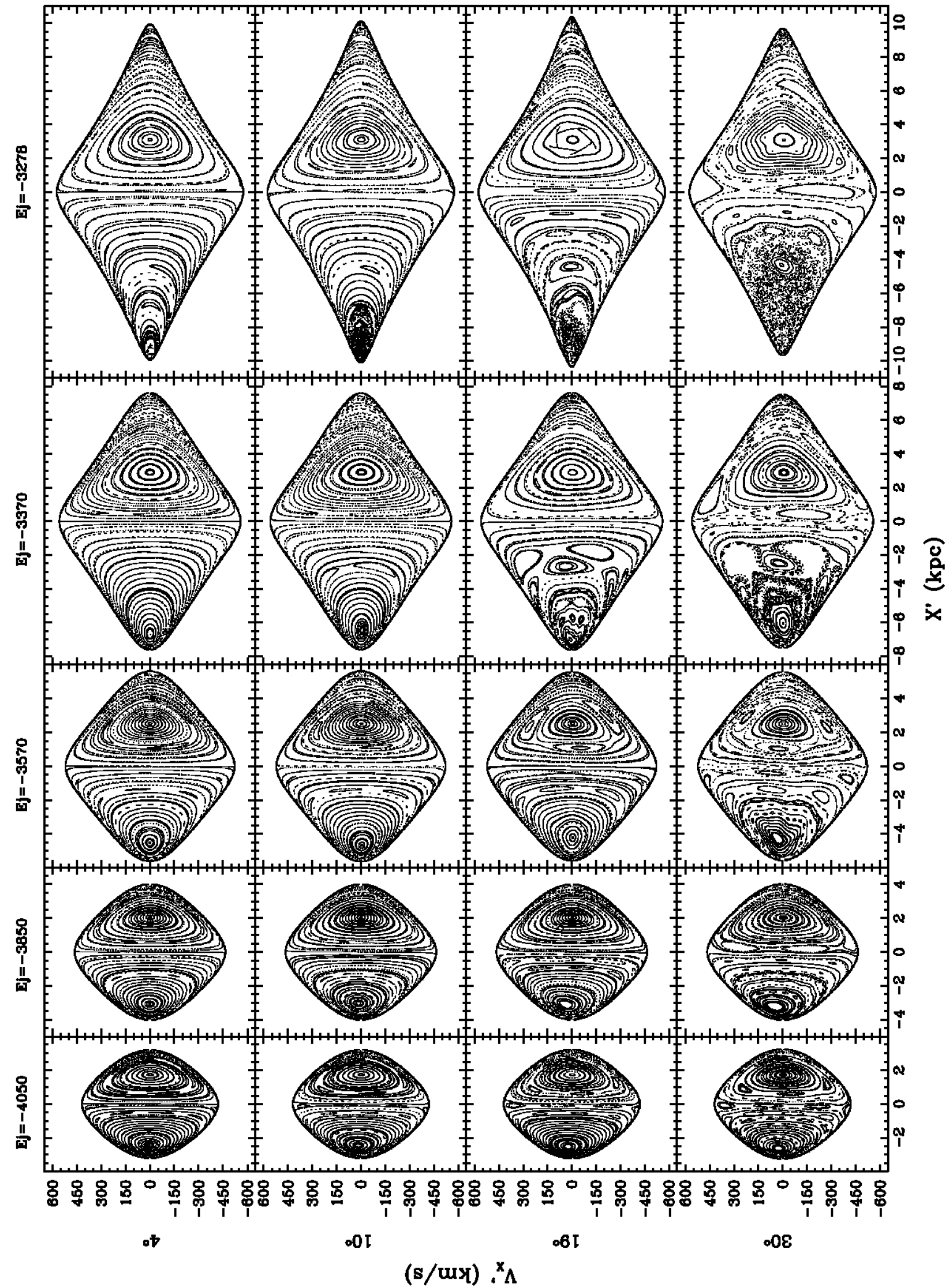

Fig. 12.- Phase space diagrams for an Sa galaxy, with $E_{J}=[-4050,-3278]$, in units of $10^{2} \mathrm{~km}^{2} \mathrm{~s}^{-2}$. From top to bottom panels of the diagram, pitch angles go from $4^{\circ}$ to $30^{\circ}$. 


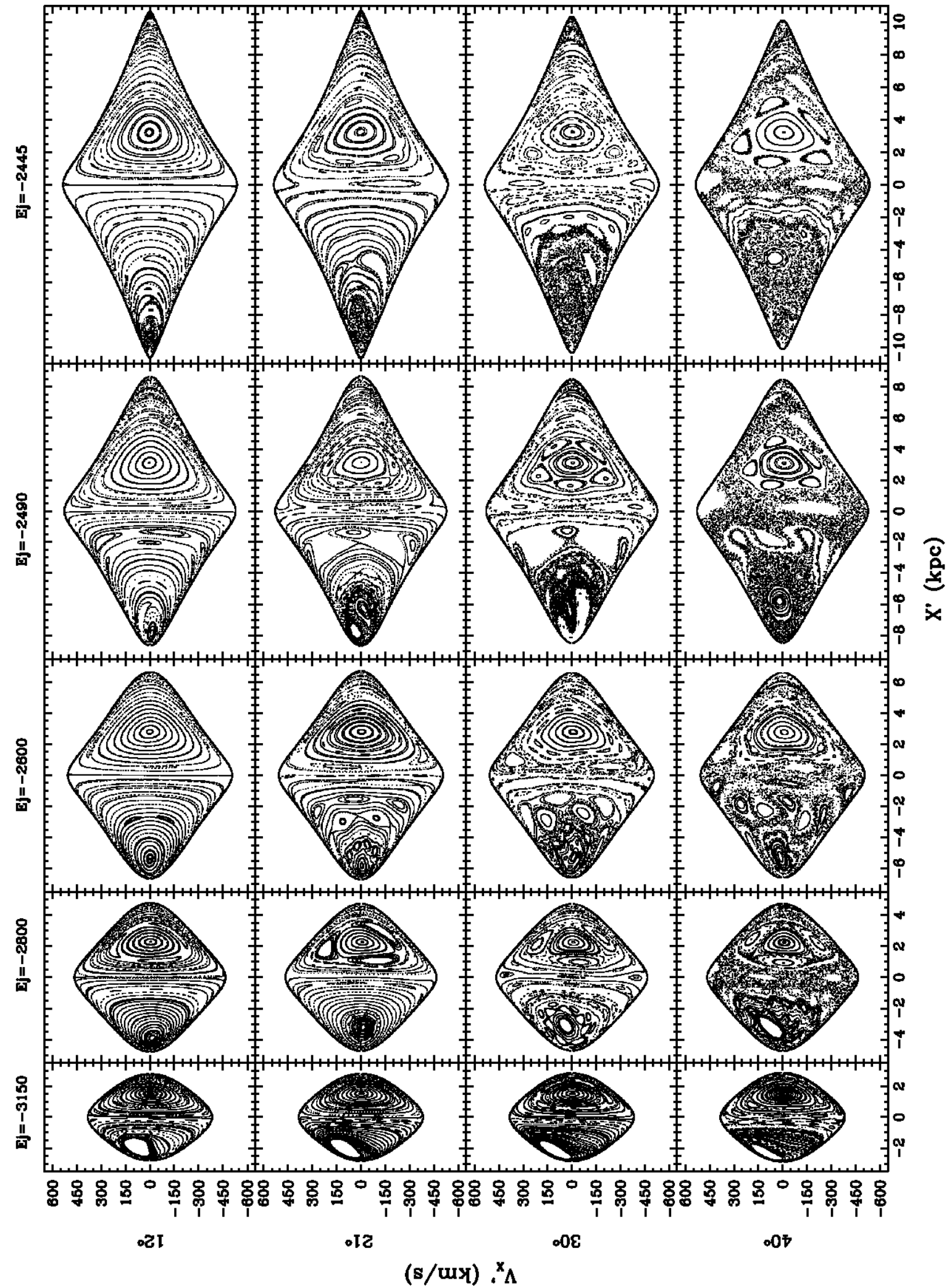

Fig. 13. - Phase space diagrams for an Sb galaxy, with $E_{J}=[-3150,-2445]$, in units of $10^{2} \mathrm{~km}^{2} \mathrm{~s}^{-2}$. From top to bottom panels of the diagram, pitch angles go from $12^{\circ}$ to $40^{\circ}$. 


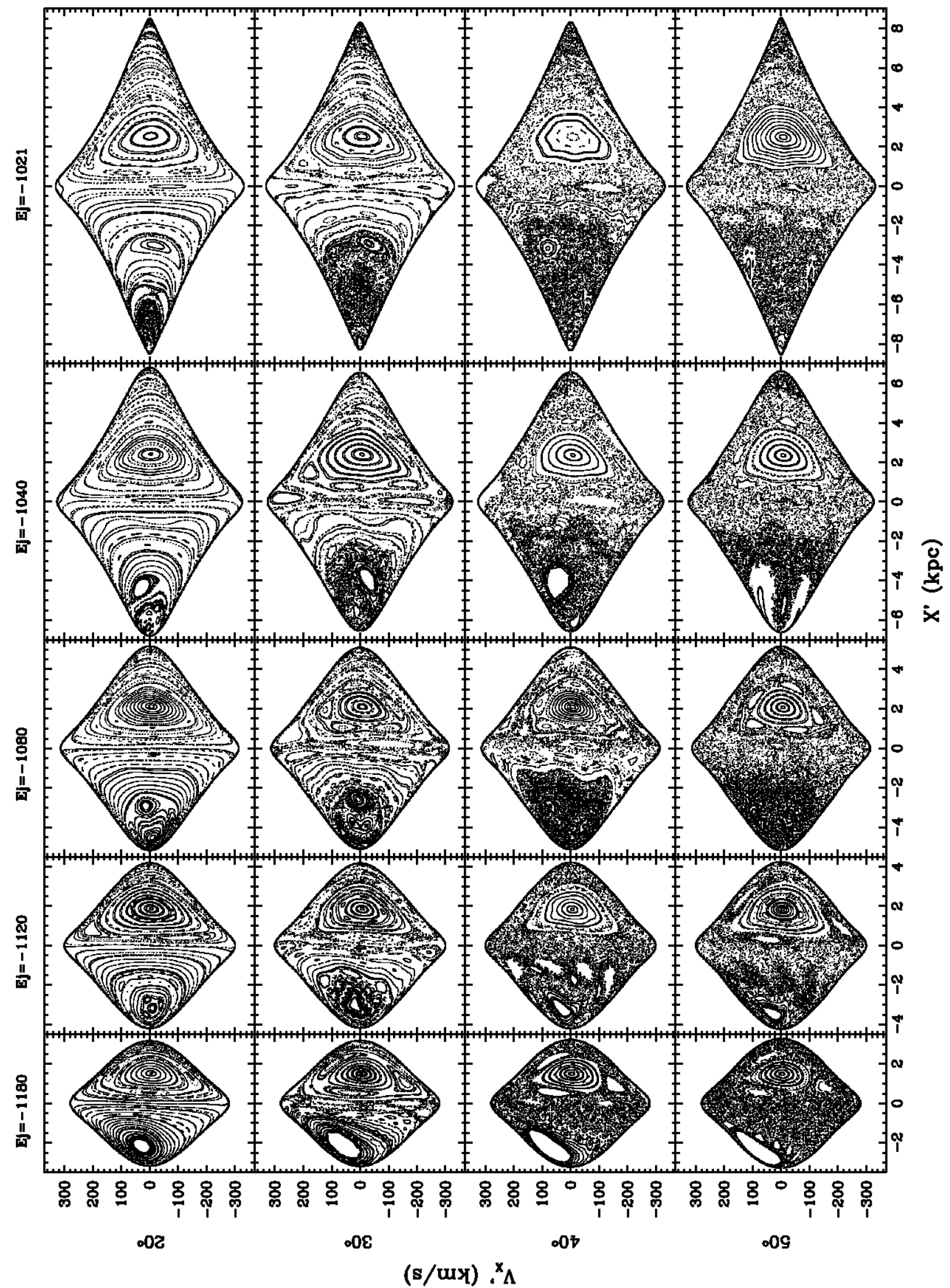

Fig. 14. - Phase space diagrams for an Sc galaxy, with $E_{J}=[-1180,-1021]$, in units of $10^{2} \mathrm{~km}^{2} \mathrm{~s}^{-2}$. From top to bottom panels, pitch angles go from $20^{\circ}$ to $50^{\circ}$. 